\title{
A 40-Gb/s 1.5- $\mathrm{mm}$ VCSEL Link with a Low-Power SiGe VCSEL Driver and TIA Operated at $2.5 \mathrm{~V}$
}

\author{
Wouter C. Soenen ${ }^{1}$, Bart Moeneclaey ${ }^{1}$, Xin $_{\text {Yin }}{ }^{1}$, Silvia Spiga $^{2}$, \\ Markus-Christian Amann ${ }^{2}$, Christian Neumeyr ${ }^{3}$, Markus Ortsiefer ${ }^{3}$, Elad Mentovich ${ }^{4}$, \\ Dimitris Apostolopoulos $^{\mathbf{5}}$, Paraskevas Bakopoulos ${ }^{\mathbf{5}}$, Johan Bauwelinck ${ }^{\mathbf{1}}$ \\ ${ }^{1}$ IDLab Dep. INTEC, Ghent University-imec, Technologiepark-Zwijnaarde 15, 9052 Gent-Zwijnaarde, Belgium \\ ${ }^{2}$ Walter Schottky Institute, Technische Universität München, Am Coulombwall 4, D-85748 Garching, Germany \\ ${ }^{3}$ Vertilas GmbH, Daimlerstr. 11d, D-85748 Garching,Germany \\ ${ }^{4}$ Mellanox Technologies, Hakidma 26, 2069200 Yokneam, Israel \\ ${ }^{5}$ Dep. Electrical \& Computer Engineering, NTUA, Iroon Polytechniou 9, 15780 Athens, Greece \\ author email address: wouter.soenen@intec.ugent.be
}

\begin{abstract}
VCSEL links typically require multiple supply voltages for high-speed and low-power operation. We report a $40-\mathrm{Gb} / \mathrm{s} 1.5-\mu \mathrm{m}$ VCSEL link achieving $8.7 \mathrm{pJ} / \mathrm{bit}$ of energy efficiency with a $0.13-\mu \mathrm{m}$ SiGe VCSEL driver and TIA operated at $2.5 \mathrm{~V}$.
\end{abstract}

OCIS codes: (250.3140) Integrated optoelectronic circuits; (250.7260) Vertical-cavity surface-emitting lasers

\section{Introduction}

High-speed vertical-cavity surface-emitting laser (VCSEL) drivers that directly modulate the laser current are available in two configurations: anode drive and cathode drive. Anode drive has the potential to lower the supply voltage of the VCSEL driver, whereas cathode drive avoids the use of the slower p-type transistors in the high-speed path. Both still have one thing in common though, namely that the VCSEL driver is operated from multiple supply voltages to lower power consumption [1-4], with laser supply voltages ranging from $3.3 \mathrm{~V}$ to $5.8 \mathrm{~V}$. In this paper, we will further focus on cathode drive and propose a solution to get rid of the multiple supply voltages.

Cathode drive VCSEL transmitters can be implemented with a back-termination resistor at the output to improve the transition times. Unfortunately, this introduces a current path between the supply voltage of the driver $V_{\text {driver }}$ and the supply voltage of the common-anode laser $V_{\text {anode }}$. This can only be nulled by choosing $V_{\text {anode }}$ equal to the sum of $V_{\text {driver }}$ and the laser forward threshold voltage $V_{l}$, enforcing the vendor to provide multiple supply voltages.

We present a $0.13 \mu \mathrm{m} \mathrm{SiGe} \mathrm{BiCMOS}$ driver and transimpedance amplifier (TIA) integrated circuit (IC) that can operate a single-mode $1.5 \mu \mathrm{m}$ VCSEL link up to $40 \mathrm{~Gb} / \mathrm{s}$ using a single supply voltage of $2.5 \mathrm{~V}$. Although the driver is equipped with a back-termination resistor $R_{t}$ of $100 \Omega$, aforementioned problems are eliminated by the balanced regulated output stage shown in Fig. 1. The output stage tracks the anode voltage $V_{\text {anode }}$ on-chip and adjusts the common node voltage $V_{z}$, through a voltage replica of the laser and a local voltage converter, to stabilize the VCSEL current $I_{v}$. A balancing circuit draws a dummy current $I_{v d}$ equal to $I_{v}$ at low frequencies in order to isolate the voltage converter dynamics from the high-speed output current. The output stage is preceded with a 2-tap feed-forward equalization (FFE) driver of which the magnitude, sign and delay difference of taps A1 and A0 can be modified.

The $0.13 \mu \mathrm{m}$ SiGe receiver used in the experiments is composed of a common-emitter shunt feedback TIA followed by a cascade of amplifiers. This design is a modified version of [5] in which the resistive shunt feedback is replaced with an active feedback circuit. The gain of the TIA and amplifier stages can be adapted for linear or limiting operation.

\section{Experiments}

The transmitter die measures $2.5 \mathrm{~mm} \times 1.3 \mathrm{~mm}$ and is designed as a 4-channel driver with a channel pitch of $300 \mu \mathrm{m}$. The driver outputs are wire bonded to two different $2 \times 1$ single-mode VCSEL arrays developed by Technische Universität München, see Fig. 1. Only the rightmost VCSEL is used for the experiments of this paper. The double-mesa short-cavity $1543 \mathrm{~nm}$ device is characterized by a buried tunnel junction diameter of $5 \mu \mathrm{m}$, a bottom-mesa diameter of $18 \mu \mathrm{m}$ and a small-signal bandwidth in excess of $19 \mathrm{GHz}$ [6]. The maximum output power of $4.5 \mathrm{~mW}$ occurs at a rollover current of $18 \mathrm{~mA}$. The receiver is a 2-channel TIA with a channel pitch of $750 \mu \mathrm{m}$. The TIA measures $3.6 \mathrm{~mm} \times 0.98 \mathrm{~mm}$ and is wire bonded to two separate photodiodes (PDs). The PD has an active area of $12 \mu \mathrm{m}$ and a responsivity of $0.63 \mathrm{~A} / \mathrm{W}$. The $\mathrm{PD}$ bandwidth is above $35 \mathrm{GHz}$ for bias voltages between $2.5 \mathrm{~V}$ and $3 \mathrm{~V}$. 

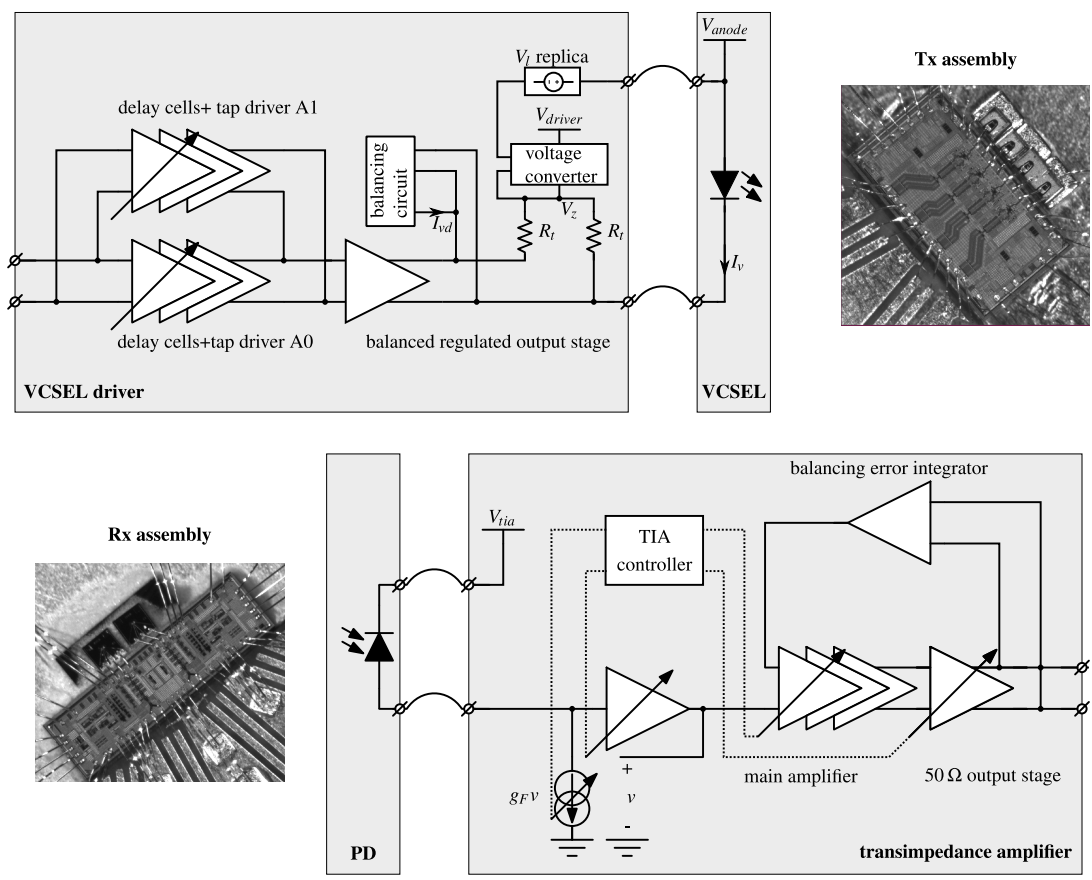

Fig. 1. Block diagram of the $0.13 \mu \mathrm{m}$ SiGe BiCMOS VCSEL driver and receiver together with a micrograph of the wire bond assembly. Supply voltages $V_{\text {driver }}, V_{\text {anode }}$ and $V_{\text {tia }}$ are all equal to $2.5 \mathrm{~V}$.

The performance of the VCSEL link is tested at 28 and $40 \mathrm{~Gb} / \mathrm{s}$ using a differential $600 \mathrm{mV}$ pseudorandom bit sequence (PRBS) of $2^{7}-1$ as input signal for the driver. Light is coupled in and out of the optical devices using lensed fibers. Sensitivity curves are measured back-to-back (BTB) and over $100 \mathrm{~m}$ and $500 \mathrm{~m}$ of single-mode fiber (SMF). The concept of the balanced regulated output stage inside the transmitter will also be verified.

\section{Results and discussion}

The VCSEL is biased at an average current of $12 \mathrm{~mA}$ for all experiments. An extinction ratio (ER) of $5.3 \mathrm{~dB}$ is achieved before equalization and reduces to $3.6 \mathrm{~dB}$ after equalization. The received eye diagrams at $28 \mathrm{~Gb} / \mathrm{s}$ and $40 \mathrm{~Gb} / \mathrm{s}$ are shown in Fig. 2 together with the bit-error ratio (BER) curves. The TIA is pushed to the limiting region to maximize the eye opening, resulting in a differential swing of $400 \mathrm{mVpp}$. Applying FFE improves the eye height at $40 \mathrm{~Gb} / \mathrm{s}$, although the performance improvement is more clear in the BER curves, revealing a gain in sensitivity of $2.3 \mathrm{~dB}$ optical modulation amplitude (OMA) at a BER below $10^{-11}$. Increasing the data rate from $28 \mathrm{~Gb} / \mathrm{s}$ to $40 \mathrm{~Gb} / \mathrm{s}$ induces a power penalty of $2 \mathrm{~dB}$ OMA. The VCSEL link is able to operate error-free, i.e. a BER of $10^{-13}$ is maintained for at least $60 \mathrm{~s}$, over $500 \mathrm{~m}$ at $28 \mathrm{~Gb} / \mathrm{s}$ and $100 \mathrm{~m}$ at $40 \mathrm{~Gb} / \mathrm{s}$. At $500 \mathrm{~m}$, the maximum optical power coupled into the PD is limited by the setup preventing to go below a BER of $10^{-9}$. The power consumed by the receiver amounts $175 \mathrm{~mW}$ while the transmitter dissipates $132 \mathrm{~mW}$ at $28 \mathrm{~Gb} / \mathrm{s}$ and $172 \mathrm{~mW}$ at $40 \mathrm{~Gb} / \mathrm{s}$, leading to $8.7 \mathrm{pJ} / \mathrm{bit}$ at $40 \mathrm{~Gb} / \mathrm{s}$. Although the link is around $5 \mathrm{~dB}$ less sensitive than [3,4], our link is almost three times more energy efficient while operating at $2.5 \mathrm{~V}$ compared to the 4,5 and $5.8 \mathrm{~V}$ used in [3]. With respect to a $25 \mathrm{~Gb} / \mathrm{s}$ common-cathode VCSEL link operating at 1 and $3.3 \mathrm{~V}$ [1], our common-anode link is $3 \mathrm{~dB}$ more sensitive at $28 \mathrm{~Gb} / \mathrm{s}$ with similar power consumption.

Figure 3 clearly shows that the transmitter can preserve a constant current through the laser over a wide range of anode voltages. The BER curves prove that high-speed performance is only marginally affected when changing the anode voltage, i.e. when the switching transistors are not pushed into saturation as is the case at $2.3 \mathrm{~V}$.

\section{Conclusion}

We have successfully demonstrated a $0.13 \mu \mathrm{m}$ SiGe driver and TIA operating a $1.5 \mu \mathrm{m}$ VCSEL link at $40 \mathrm{~Gb} / \mathrm{s}$ errorfree over $100 \mathrm{~m}$ of SMF. Most notably, these results are obtained running the ICs of a single supply voltage of $2.5 \mathrm{~V}$ while achieving an energy efficiency of $8.7 \mathrm{pJ} / \mathrm{bit}$. 

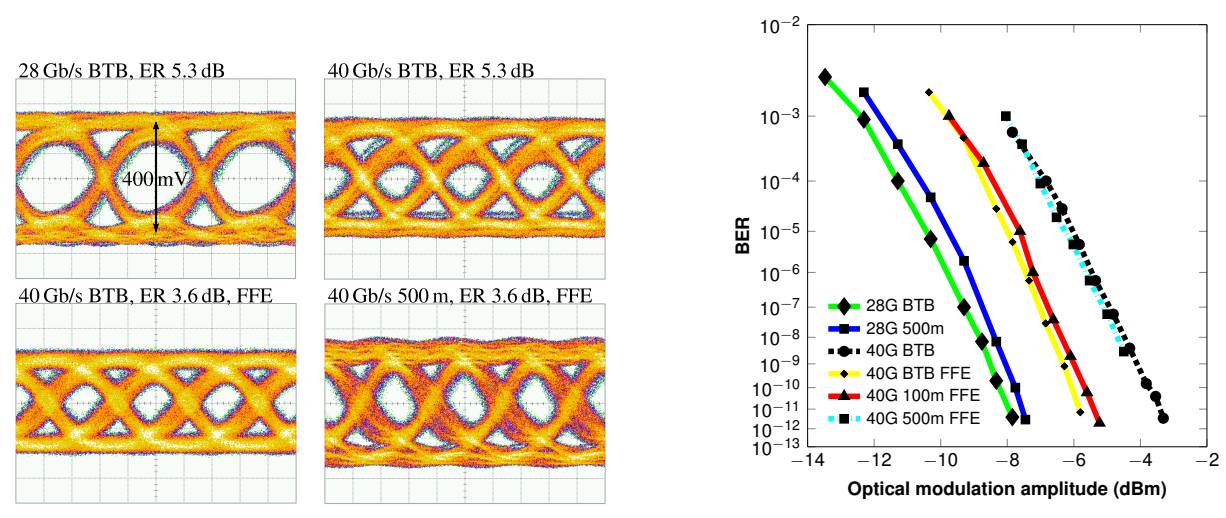

Fig. 2. Received eye diagrams and BER curves at $28 \mathrm{~Gb} / \mathrm{s}$ and $40 \mathrm{~Gb} / \mathrm{s}$ for various transmission distances. Remark that the eye diagram at $500 \mathrm{~m}$ was recorded at a higher input power.
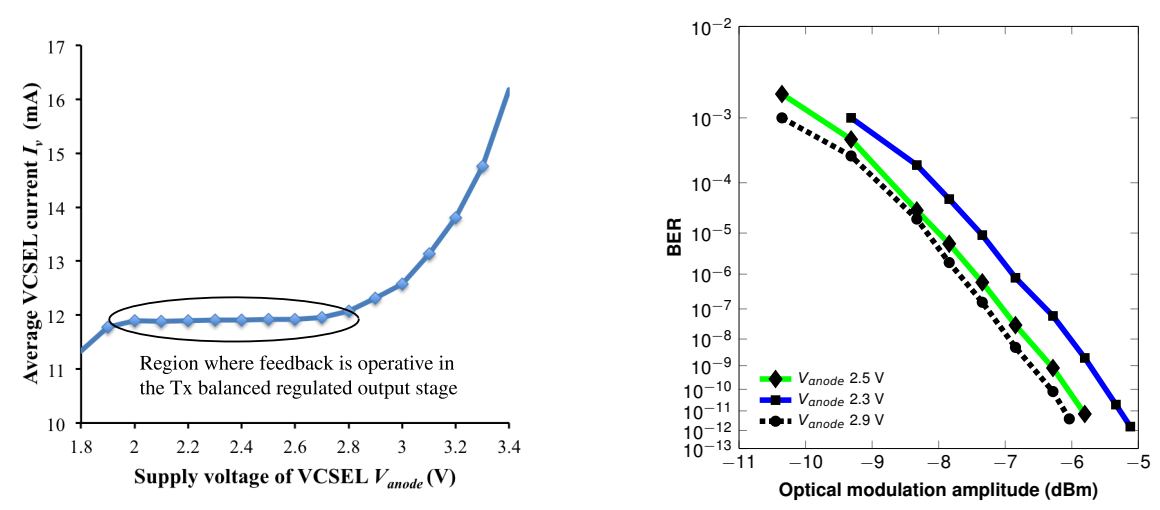

Fig. 3. Impact of the VCSELs anode voltage at DC and at $40 \mathrm{~Gb} / \mathrm{s}$

\section{Acknowledgments}

The authors would like to thank Albis for their photodiodes and imec and Anna Sandomirsky from Mellanox for wire bonding the assemblies. This work was supported by the European FP7 projects Mirage and Phoxtrot.

\section{References}

1. T. Yazaki, N. Chujo, H. Yamashita, T. Takemoto, Y. Lee and Y. Matsuoka, "25-Gbps x 4 optical transmitter with adjustable asymmetric pre-emphasis in 65-nm CMOS," in 2014 IEEE International Symposium on Circuits and Systems (ISCAS), Melbourne VIC, 2014, pp. 2692-2695.

2. Y. Tsunoda, M. Sugawara, H. Oku, S. Ide and K. Tanaka, " 8.9 A 40Gb/s VCSEL over-driving IC with groupdelay-tunable pre-emphasis for optical interconnection," in 2014 IEEE International Solid-State Circuits Conference Digest of Technical Papers (ISSCC), San Francisco, CA, 2014, pp. 154-155.

3. A. V. Rylyakov et al., "A 40-Gb/s, 850-nm, VCSEL-based full optical link," in Optical Fiber Communication Conference and Exposition (OFC/NFOEC), 2012 and the National Fiber Optic Engineers Conference, Los Angeles, CA, 2012, pp. 1-3.

4. D. Kuchta et al., "Error-free $56 \mathrm{~Gb} / \mathrm{s}$ NRZ Modulation of a $1530 \mathrm{~nm}$ VCSEL Link," J. Light. Technol., vol. 34, no. 14, pp. 3275-3282, July 2016.

5. B. Moeneclaey et al., "A 40-Gb/s Transimpedance Amplifier for Optical Links," in IEEE Photonics Technology Letters, vol. 27, no. 13, pp. 1375-1378, July 2015.

6. S. Spiga, D. Schoke, A. Andrejew, G. Boehm and M. C. Amann, "Enhancing the small-signal bandwidth of single-mode 1.5- $\mu \mathrm{m}$ VCSELs," in IEEE Optical Interconnects Conference, San Diego, CA, 2016, pp. 14-15. 